\title{
Integration, heterochrony, and adaptation in pedal digits of syndactylous marsupials Vera Weisbecker*1 and Maria Nilsson²
}

\author{
Address: ${ }^{1}$ School of Biological, Earth and Environmental Sciences, University of New South Wales, UNSW/Sydney, NSW 2052, Australia and 2 Lund \\ University, Department of Cell and Organism Biology, Genetics, Division of Evolutionary Molecular Systematics, Sölvegatan 29 , S-223 62 Lund, \\ Sweden \\ Email: Vera Weisbecker* - v.weisbecker@student.unsw.edu.au; Maria Nilsson - maria.nilsson@cob.lu.se \\ * Corresponding author
}

Published: 25 May 2008

BMC Evolutionary Biology 2008, 8:160 doi:10.1/86/147|-2148-8-160
Received: 2I February 2008

Accepted: 25 May 2008

This article is available from: http://www.biomedcentral.com/147/-2/48/8//60

(C) 2008 Weisbecker and Nilsson; licensee BioMed Central Ltd.

This is an Open Access article distributed under the terms of the Creative Commons Attribution License (http://creativecommons.org/licenses/by/2.0), which permits unrestricted use, distribution, and reproduction in any medium, provided the original work is properly cited.

\begin{abstract}
Background: Marsupial syndactyly is a curious morphology of the foot found in all species of diprotodontian and peramelemorph marsupials. It is traditionally defined as a condition in which digits II and III of the foot are bound by skin and are reduced. Past treatments of marsupial syndactyly have not considered the implications of this unique morphology for broader issues of digit development and evolution, and the ongoing debate regarding its phylogenetic meaning lacks a broad empirical basis. This study undertakes the first interdisciplinary characterisation of syndactyly, using variance/covariance matrix comparisons of morphometric measurements, locomotor indices, ossification sequences, and re-assessment of the largely anecdotal data on the phylogenetic distribution of tarsal/metatarsal articulations and "incipient syndactyly".

Results: Syndactylous digits have virtually identical variance/covariance matrices and display heterochronic ossification timing with respect to digits IV/V. However, this does not impact on overall locomotor adaptation patterns in the syndactylous foot as determined by analysis of locomotor predictor ratios. Reports of incipient syndactyly in some marsupial clades could not be confirmed; contrary to previous claims, syndactyly does not appear to impact on tarsal bone arrangement.

Conclusion: The results suggest that marsupial syndactyly originates from a constraint that is rooted in early digit ontogeny and results in evolution of the syndactylous digits as a highly integrated unit. Although convergent evolution appears likely, syndactyly in Diprotodontia and Peramelemorpha may occur through homologous developmental processes. We argue that the term "syndactyly" is a misnomer because the marsupial condition only superficially resembles its name-giving human soft-tissue syndactyly.
\end{abstract}

\section{Background}

The occurrence of homoplasy is problematic in phylogenetic reconstruction and the tracing of morphological evolution $[1,2]$. This is particularly the case when the evolution of a homoplastic trait is perceived as relatively com- plex and therefore deemed unlikely to have evolved more than once. A classic example that has sustained nearly two centuries of controversial and often heated debate is the character of marsupial syndactyly. This is commonly defined as a peculiar phenotype in which digits II and III 
of the marsupial foot are tightly connected by a common sheath of skin at least to the base of the intermediate phalanx $[3,4]$. The trait occurs in all species of two Australasian marsupial orders (Fig. 1), Peramelemorpha (bilbies and bandicoots; 21 species) and Diprotodontia (koalas, wombats, possums, kangaroos, and allies; approx. 141 species). Together, these two clades represent nearly 50\% of marsupial species [5], Diprotodontia being the most ecologically and locomotorily diverse extant marsupial clade $[6,7]$.

Marsupial syndactyly was one of the first characters used for marsupial classification, [8-12]. It has been reasoned that a character as complex as syndactyly could have only evolved once within marsupials $[8,12-16]$, and peramelemorphs and diprotodontians were commonly grouped in the clade of Syndactyla $[11,16,17]$. Famously, this was at odds with the division of marsupials based on dentition into "diprotodont" (including Diprotodontia, and sometimes Paucituberculata, which possess two large procumbent incisors) and "polyprotodont" clades [peramelemorphs and all other marsupial clades $[6,10,18]]$. However, with the advent of molecular systematics, it became widely accepted [with some exceptions; $[16,19]]$ that Diprotodontia and Peramelemorpha are not sister groups [20-23]. As Figure 1 shows, this arrangement favours homoplastic origins of syndactyly in Peramelemorpha and Diprotodontia [18,21,23-25].

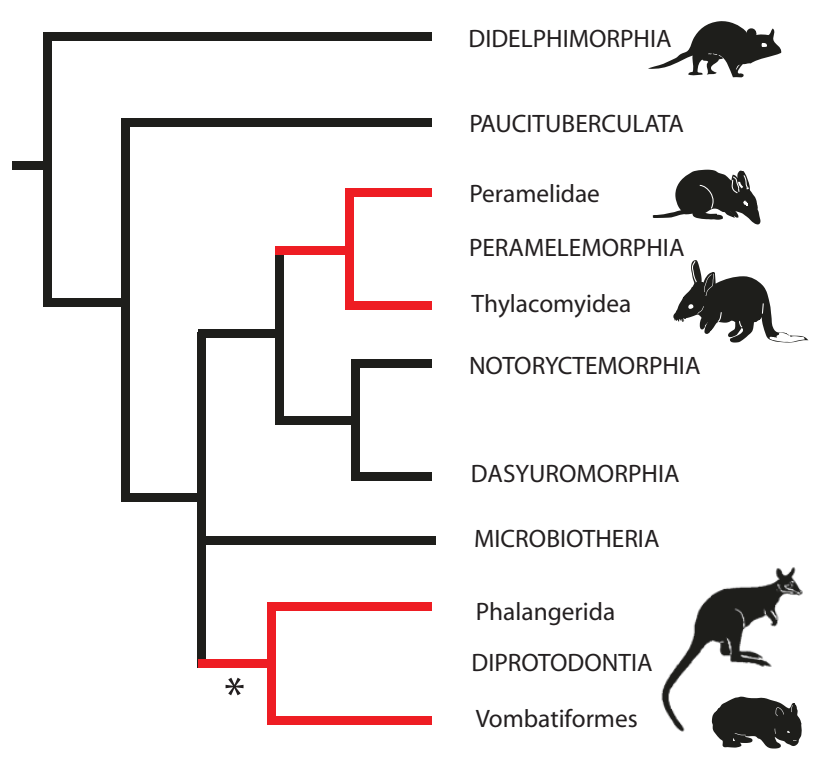

\section{Figure I}

Phylogeny of marsupial orders. Figure based on a consensus of Amrine-Madsen et al. (2003) and Nilsson et al. (2004), with unresolved position of Microbiotheria. Red branches denote clades where syndactyly occurs.
However, there have also been suggestions that syndactyly may be plesiomorphic for larger marsupial clades, and has subsequently been lost in some clades $[23,26,27]$.

As a matter of course, it has been assumed that marsupial syndactyly arose under selective pressure, but what the functional relevance of syndactyly is has never been convincingly argued. A number of researchers suggested that the comparatively small size ("reduction") of syndactylous digits in most species is related to arboreal locomotion in putatively arboreal ancestors of the syndactylous clades $[13,28,29]$. This scenario has been contested because arboreal Diprotodontia have particularly strongly developed syndactylous digits $[3,30]$. A second hypothesis suggests that the function of syndactylous digits as "grooming digits" confers a selective advantage $[3,11,18,31]$. However, grooming organs are common among placental mammals without comparable extensive transformations in pedal morphology $[3,13]$. As such, the adaptive implications of marsupial syndactyly remain elusive. However, it has never been considered that it is a unique phenomenon with implications transcending its local importance. No extant tetrapod clade displays a trait which, without a clear-cut locomotor function, impacts as heavily and consistently on autopodial morphology of an ecologically diverse order. Such exceptions have the potential to inform our understanding of evolutionary and developmental patterns of tetrapod autopodial evolution [32], but little empirical information exists on syndactyly, which stems mainly from a few older dissectionbased studies [3,33-35]. As such, syndactyly displays some intriguing but largely unexplored characteristics. For example, syndactyly is not an exclusively soft-tissue related condition but majorly affects the digits (Fig. 2). The characteristic morphology of digits II and III suggests that they evolve as a distinct unit within the syndactylous foot [4], but this has never been tested. The result of the unusual anatomy of syndactylous digits is that digit III never forms the main axis of the foot in syndactylous marsupials [36], which is rare among tetrapods [37,38]. Instead, the marginal digits IV and $\mathrm{V}$ are extensively developed, while digits II and III are considered "reduced" $[13,31]$. Nevertheless, locomotion in syndactylous marsupials (particularly Diprotodontia) is more diverse than in all other marsupials and many placental orders, catering for plantigrade terrestrial walking and bounding, grasping arboreal locomotion, and the unique hopping gait of kangaroos; this is also reflected in pedal diversity (Fig. 2). However, patterns of functional adaptation in the syndactylous foot and particularly of digits II and III remain to be explored. Marsupial syndactyly is also apparent very early in pedal development, which has lead to the as yet untested suggestion of heterochronic change of foot development in syndactylous species [13]. This is an interesting suggestion because heterochronic change in early 
ontogeny can help to explain evolutionary transformations of morphological traits [39-41]. Lastly, contextualisation of syndactyly in an evolutionary framework is to date impossible because the evidence regarding its distribution has not been comprehensively assessed; two lines of evidence have been deemed important in this respect. A range of didelphid species have been termed "incipiently syndactylous" (Table 1). This has been considered evidence that syndactyly may be more plesiomorphic within marsupials than has been generally recognized $[23,26,42]$, although the existence of incipient syndactyly has been contested $[30,42,43]$. Contrary to this hypothesis, it was suggested that tarsal/metatarsal articulation patterns in peramelemorphs, which differ from the plesiomorphic mammalian condition, are a consequence of convergent acquisition of syndactyly $[8,15]$.

\section{Characterizing Syndactyly}

This study approaches marsupial syndactyly from several angles. First, we test the notion that digits II and III evolve as a distinct unit within the syndactylous foot. This is done through assessment of inter-digit integration patterns of morphometric measurements across the diversity of syndactylous marsupials, using a comparison of variance/covariance and correlation matrices. A morphometric approach is also taken to explore the impact of marsupial syndactyly on pedal diversity and functional adaptation. In addition, comparative analysis of ossification sequence in the digits [44-46] is employed to assess the proposition that syndactyly arises through heterochronic change in pedal digit development [13]. Lastly, a review and acquisition of new broad-scale comparative data on external didelphimorph pedal digit morphology, as well as marsupial tarsal articulations, are employed for empirical qualification of previous hypotheses regarding incipient syndactyly and the influence of syndactyly on tarsal morphology.

Using this combination of morphometric, external morphological, and ontogenetic approaches, we address issues regarding the nature, origins, and evolutionary implications of marsupial syndactyly: What are the evolu- tionary characteristics of digit relationships in syndactylous marsupials? Are these patterns related to heterochronic changes in pedal digit development? Does syndactyly impact on pedal diversity and functional adaptation? Does a reassessment of traditionally used indicators of syndactyly result in a better understanding of its evolution? The results are synthesized into an integrated view of marsupial syndactyly as a unique phenomenon in tetrapod autopodial evolution.

\section{Results \\ Correlation matrix comparisons}

Morphometric raw data are shown in Additional file 1. Variance/Covariance (v/cv) and correlation matrix correlations are listed in Table 2. Matrix repeatabilities (listed in the diagonals of Table 2) are mostly over 0.9, suggesting low sampling error. One exception are the repeatabilities for $\mathrm{v} / \mathrm{cv}$ matrices of digits II and III which are considerably lower (this is probably due to size variation, which is greater than in the remaining digits). This is likely an underestimate since the lower repeatabilities for these digits lead to over-adjustment of their raw v/cv matrix correlations (which is very high; 0.96 ) to a correlation value over 1 . The highest matrix correlations reported by both analyses are those between digits II and III. Medium to high correlations are reported for comparisons of digit $\mathrm{V}$ with all other digits, while matrices of digits IV compared with digits II and III have the lowest correlations. Variance/covariance matrix correlations are consistently higher that correlation matrix comparisons, except in comparisons between the syndactylous digits. This difference might be due to the scaling effect on the non-size adjusted data resulting from division by the standard deviation in the correlation approach [47].

The correlations between raw lengths are significantly stronger between phalangeal elements of digits II and III compared to those of digits II or III with digits IV or $\mathrm{V}$ (Table 3). Regressions of raw measurements of corresponding digit elements show that this is due to a virtually isometric relationship between digits II and III as evidenced by regression slopes close to 1 , with very low

Table I: List of species considered incipiently syndactylous

\begin{tabular}{ll}
\hline Species & Author \\
\hline Caluromys derbianus & Kirsch 1977, Bensley 1903 \\
Genus Marmosa & Bensley 1903, Tate 1933, Kirsch 1977 \\
Monodelphis orinoci & Kirsch 1977 \\
Thylamys pusilla & Bensley 1903 \\
Gracilianus microtarsus & Bensley 1903 \\
Philander opossum & Bensley 1901 \\
Chironectes minimus & Hall I987 \\
Notoryctes typhlops & Gadow 1892, Dollo 1899, Bensley 1903, Szalay 1982, 1993, I994 \\
Genus Micoureus & Bensley 1901 \\
\hline
\end{tabular}




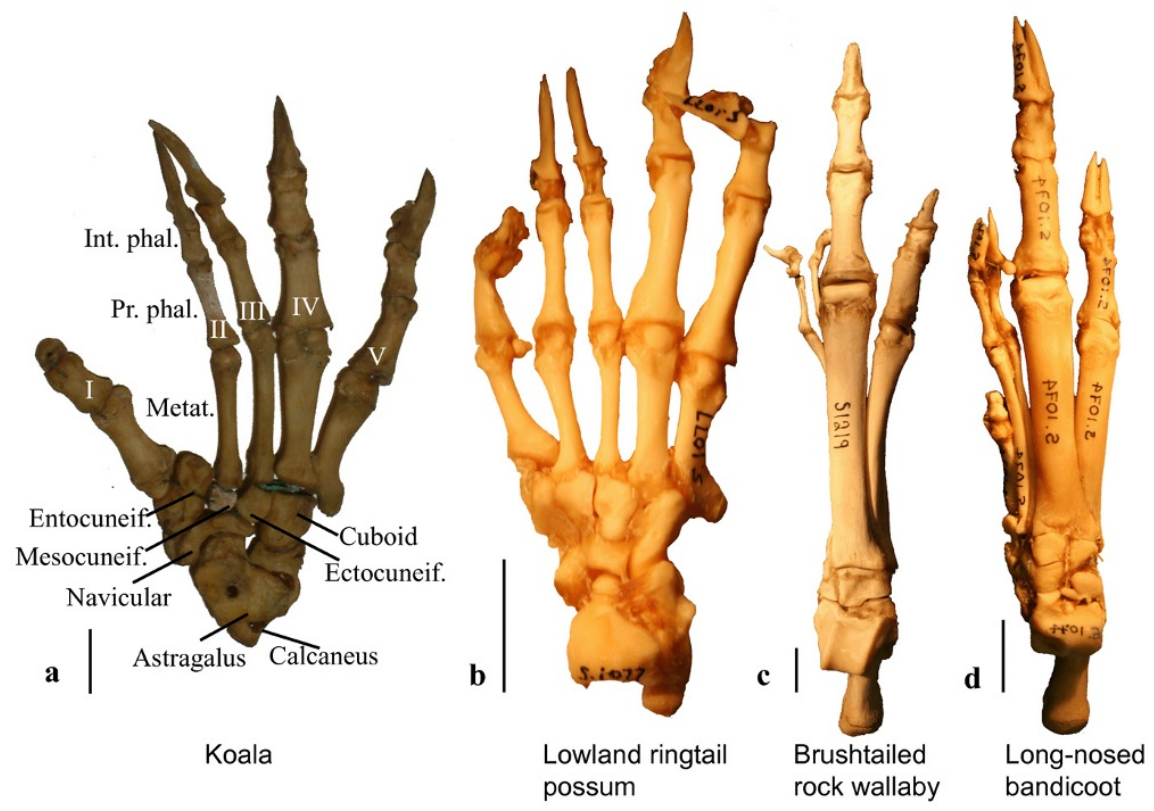

\section{Figure 2}

Syndactylous feet. Explanation of terminology and images of the right pedal skeleton of four syndactylous marsupials, showing tarsal bones and separated digits of a koala (a), a lowland ringtail possum Pseudochirulus canescens (b), a brush-tailed rock wallaby (c), and a long-nosed bandicoot Perameles nasuta (d). Note that a-c are diprotodontian marsupials, while $d$ is a peramelemorph. Int. phal, intermediate phalanx; Metat., metatarsal; Prox. Phal, proximal phalanx. Roman numerals refer do digit numbers. Scale bar $=\mathrm{I} \mathrm{cm}$.

regression errors. Same-element regressions between all other digit combinations are considerably different in slope, with larger errors (Table 3; for some examples, see Fig. 3).

\section{Locomotor indices}

The Kruskal-Wallis tests of single-digit comparisons suggest highly significant differences between all locomotor types and all predictor ratios (Additional file 2). The post hoc Wilcoxon tests are generally significant between all pairwise group comparisons. The results suggest that

Table 2: Variance/Covariance and correlation matrix correlations between digits. Matrix repeatabilities are on the diagonal of the matrix.

\begin{tabular}{ccccc}
\hline Variance/covariance & II & III & IV & V \\
\cline { 2 - 5 } II & 0.78 & & & \\
III & I.18 & 0.83 & & \\
IV & 0.65 & 0.74 & 0.98 & \\
V & 0.90 & 0.95 & 0.97 & 0.97 \\
\hline Correlation & & & & \\
II & 0.99 & & & \\
III & 1.00 & 0.98 & & \\
IV & 0.60 & 0.61 & 0.91 & \\
V & 0.87 & 0.89 & 0.81 & 0.96 \\
\hline
\end{tabular}

arboreal species have longer and more gracile phalanges (see also Figure 2a, b), as demonstrated by higher phalangeal indices, metatarsal slenderness ratios (SRs), distal SRs, and proximal SRs of digits IV and V compared to quadrupedal terrestrial ones. Metatarsal SRs are highest in peramelemorphs and macropodoid species, reflecting their elongate metatarsals (see also Figs. 2c, d).

Kruskal-Wallis tests of locomotor index ratios in digit pairs shows that most between-digit relationships are significantly different between locomotor types. Exceptions are ratios between digits II and III, and all between-metatarsal ratios. The follow-up Wilcoxon test (Additional file 2 ) is significant for most inter-digit relationships between all three groups, reflecting the markedly different relative digit lengths in species of different locomotor type.

\section{Digit ossification sequences}

Ossification ranks for each species are presented in Additional file 3. In non-syndactylous marsupials, phalangeal elements of one row (e.g. proximal, intermediate) occur simultaneously in digits II-IV (Fig. 4a). In syndactylous marsupials (Fig. 4b), the majority of phalangeal row ossification sequences are disassociated in time: digit IV is earliest to ossify in a row, in most cases simultaneous with or followed by digit V. Then follow ossification of digits II 
Table 3: Raw data regression results

\begin{tabular}{|c|c|c|c|c|c|c|c|}
\hline & \multicolumn{4}{|c|}{ Regression } & \multicolumn{3}{|c|}{ Correlation comparisons between II/III and others } \\
\hline & Intercept & Error & Slope & Error & r & $\mathrm{dz}$ & $p$ \\
\hline \multicolumn{8}{|l|}{ Metatarsals } \\
\hline II and III & 0.333 & 0.924 & 0.907 & 0.028 & 0.986 & & \\
\hline II and IV & 1.167 & 1.451 & 0.766 & 0.038 & 0.965 & 1.790 & 0.074 \\
\hline II and V & 4.103 & 2.421 & 0.746 & 0.069 & 0.892 & 3.931 & 0.000 \\
\hline $\mathrm{IV}$ and $\mathrm{V}$ & 2.224 & 1.422 & 1.029 & 0.040 & 0.978 & 0.853 & 0.394 \\
\hline \multicolumn{8}{|c|}{ Pr. Phalanges } \\
\hline II and III & -0.308 & 0.210 & 1.014 & 0.016 & 0.996 & & \\
\hline II and IV & -0.886 & 1.777 & 0.732 & 0.096 & 0.812 & 7.395 & 0.000 \\
\hline $\mathrm{II}$ and $\mathrm{V}$ & -1.264 & 1.722 & 0.968 & 0.120 & 0.828 & 7.128 & 0.000 \\
\hline $\mathrm{IV}$ and $\mathrm{V}$ & 2.659 & 1.892 & 1.077 & 0.132 & 0.831 & 7.170 & 0.000 \\
\hline \multicolumn{8}{|c|}{ Int. Phalanges } \\
\hline II and III & -0.552 & 0.352 & 1.087 & 0.043 & 0.977 & & \\
\hline II and IV & -1.314 & 1.843 & 0.729 & 0.160 & $0.64 I$ & 5.57 & 0.000 \\
\hline $\mathrm{II}$ and $\mathrm{V}$ & -2.208 & 1.390 & 1.073 & 0.155 & 0.784 & 4.41 & 0.000 \\
\hline IV and V & 2.866 & 1.212 & 0.948 & 0.788 & 0.788 & 4.340 & 0.000 \\
\hline
\end{tabular}

Regression results of raw length regressions between corresponding digit elements. Note that elements of digits II and III are close to identical in length in all species as shown by intercepts near 0 and slopes close to I with very little error. This is also reflected in tight correlations between digits II and III, which are nearly all significantly stronger than any other between-digit correlations (the relatively weaker results in metatarsals comparisons are caused by a single high-leverage outlier, Diprotodon optatum). r, Pearson correlation coefficient; dz, difference of z-score transformed correlations; $p$, significance.

and III (Fig. 4b). Ossification onsets for digit I are slightly more variable, and metatarsals ossify at the same time in all species except for Cercartetus concinnus (Fig. 4b). Parsimov analysis of the ossification data, using the consensus of ACCTRAN and DELTRAN optimized apomorphy list inputs, identifies two heterochronic shifts responsible for the rank changes: firstly, an acceleration of intermediate phalanges of digits IV and $\mathrm{V}$ with respect to proximal and intermediate phalanges of digits II and III (see Fig. 5 for an example), and secondly, acceleration of distal phalanges IV and V with respect to distal phalanges II and III.

\section{Tarsal bone anatomy and "incipient syndactyly"}

All marsupial species examined, except peramelemorphs, show metatarsal-tarsal articulations that correspond to the plesiomorphic mammalian pattern (Fig. 2a-c). Digits II and III articulate with the mesocuneiform and ectocuneiform bone, respectively, in all marsupials, while in peramelemorphs, digit III articulates perceptibly less with the ectocuneiform. Digit IV is mostly supported by the cuboid in all species except peramelemorphs, where it is supported partially by the ectocuneiform and cuboid bones (Fig. 2d). However, in wombats and a range of macropodoids there is also considerable contact between digit IV and the ectocuneiform (Fig. 2c). Within peramelemorphs, there is some variation as to the degree to which the ectocuneiform is included in support of digit IV. This seems to be related to locomotor mode, as the most extensive contact is in the cursorial bilbies, Macrotis lagotis and the extinct Chaeropus ecaudatus. Digit V is supported by the cuboid in all species.

Species for which claims of "incipient syndactyly" were found in the literature are listed in Table 1. Visual investigation of study skins of didelphimorph marsupials show that in most species, all middle digits (digits II-IV) are highly similar. In some didelphimorphs, digits II and III are more gracile than digits IV and $\mathrm{V}$, and more similar to each other (for an example, see Fig. 6), but never as distinctly so as in syndactylous marsupials. In no case are digits II and III fully webbed, except in the fully webbed feet of Chironectes minimus and in the foot of Notoryctes typhlops, whose digits I-IV are webbed.

\section{Discussion}

The origins and evolution of marsupial syndactyly have been debated for more than 150 years, although little observational information has been collected. It may be that the distinctive overall anatomy of syndactyly resulted in the perception that it is distinctive enough not to require further research. However, the results of this study indicate that marsupial syndactyly has a unique evolutionary and developmental background which has previously been largely ignored. A synthesis of results from this study takes the implications of this unique phenomenon beyond its traditional role as a purely phylogenetic problem. 

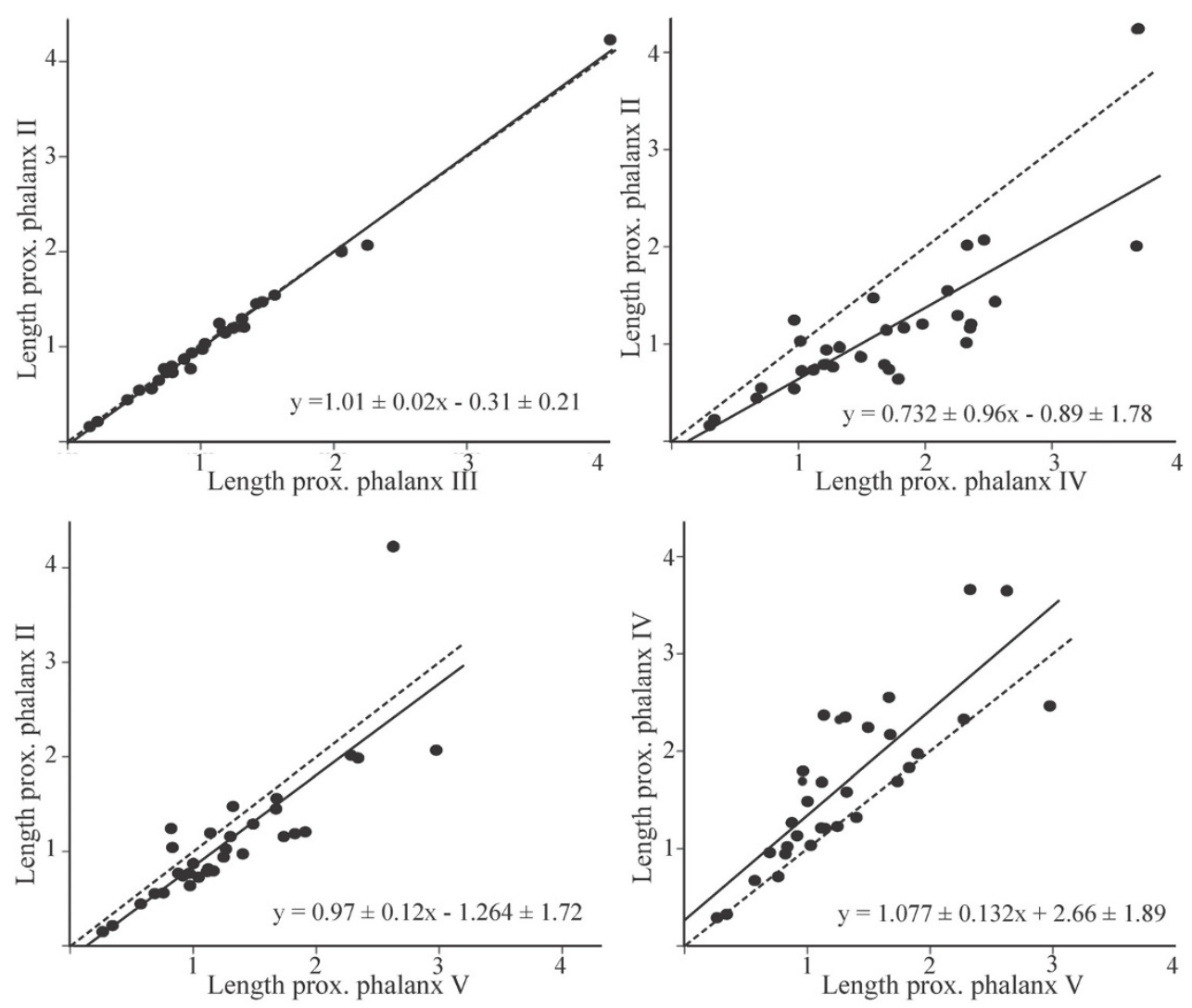

\section{Figure 3}

Raw data regressions. Representative regression plots of proximal phalangeal length of different digits. Proximal phalanges of digits II and III are virtually isometric with very little error, whereas all others, although sometimes close to isometric, have considerably larger error around the regression line.

\section{Unique integration in the syndactylous foot}

The near-identity of correlation matrices of digits II and III, combined with strict raw measurement isometry of their digit elements across species, suggest that the syndactylous digits are highly constrained with respect to each other across the diversity of syndactylous species. This is congruent with the fact that the phalangeal skeleton of the syndactylous digits is visually undistinguishable. This is reminiscent of a definition of identical digits by Tabin [[48], p. 290]: " Empirically, a digit is considered to have the same identity as a second digit ' $x$ ' if, when examined in isolation by a morphologist, the first digit would be labeled as being a digit ' $\mathrm{x}$ '". Such a condition has few counterparts in extant tetrapods. Among cases of marsupial-like syndactyly reported in placentals, only that in digit III and IV in three closely related species of otter shrew (Potamogale velox and two species of Micropotamogale) is directly comparable [49]; note that the reportedly syndactylous siamang gibbon Symphalangus syndactylus has digits of different length [50]. To our knowledge, conditions resembling marsupial syndactyly are also unknown in extant amphibians or sauropsids [51]. It could be argued that digits III and IV of hoofed placentals (artiodactyla and perissodactyla) could be comparable in their similarity to marsupial syndactyly. However, autopodia of hoofed placentals follow a welldocumented pattern of increased central digit emphasis in fore-and hindlimbs which is restricted to cursorial mammals [32,52,53]. Marsupial syndactyly is not comparable since it is restricted to the foot and morphologically and functionally diverse, and does not correspond to any obvious locomotor adaptation.

\section{Syndactyly does not constrain functional adaptation of the foot}

Autopodial anatomy in tetrapods is strongly tied to locomotor function [54-56], and this is reflected in greater variation in autopodial anatomy compared to more proximally situated limb elements [57,58]. Extensive locomotor adaptation within pedal digits is evident from 

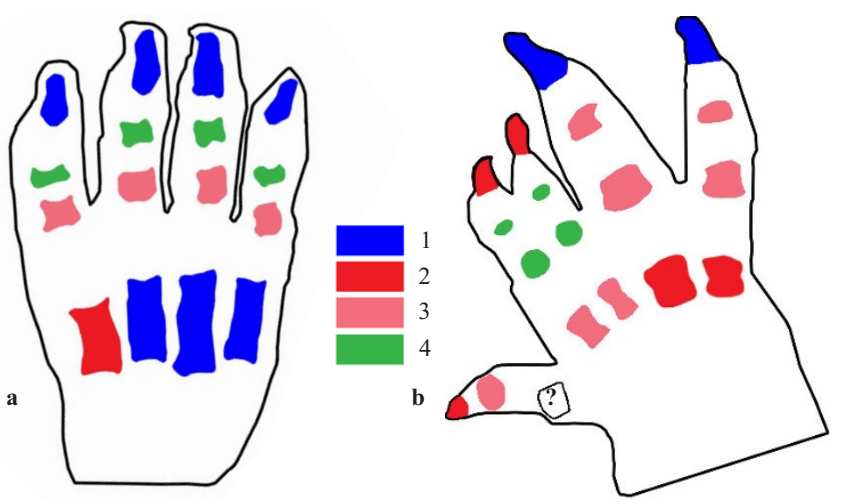

Figure 4

Pedal digit ossification in syndactylous and non-syndactylous marsupials. Schematic drawing of ossifications in the foot of a non-syndactylous pouch young Quoll ("native cat") Dasyurus viverrinus and syndactylous pygmy possum Cercartetus concinnus Elements are coloured according to the sequence in which they ossify, from first (I) to last (4). Not to scale.

the matrix comparisons involving digits II or III versus IV and V. Digit and phalangeal proportions correspond with those generally predicted for mammalian autopodia [7,55,56,59,60]: arboreal species have higher phalangeal indices and phalangeal slenderness ratios, while species with exclusively terrestrial locomotion have shorter phalanges and more sturdy phalangeal proportions.

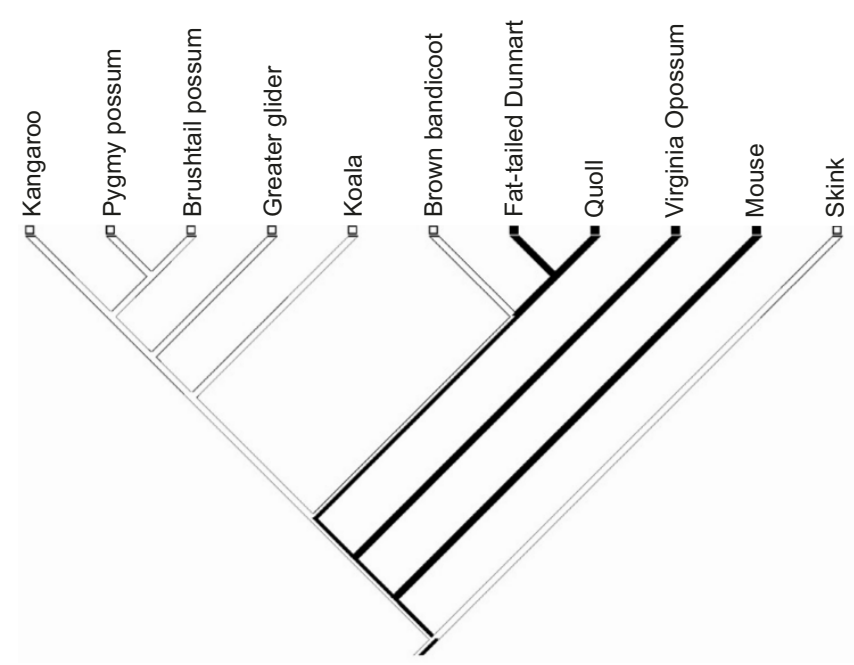

\section{Figure 5}

Event pair mapping. Phylogeny of the species used for ossification analysis of digit ossifications, with mapping of the example of scores for intermediate digit IV versus proximal digit II. In all syndactylous marsupials, intermediate digit IV ossifies before proximal digit II (white lines), while both ossifications appear simultaneously in all other mammals (solid black line).

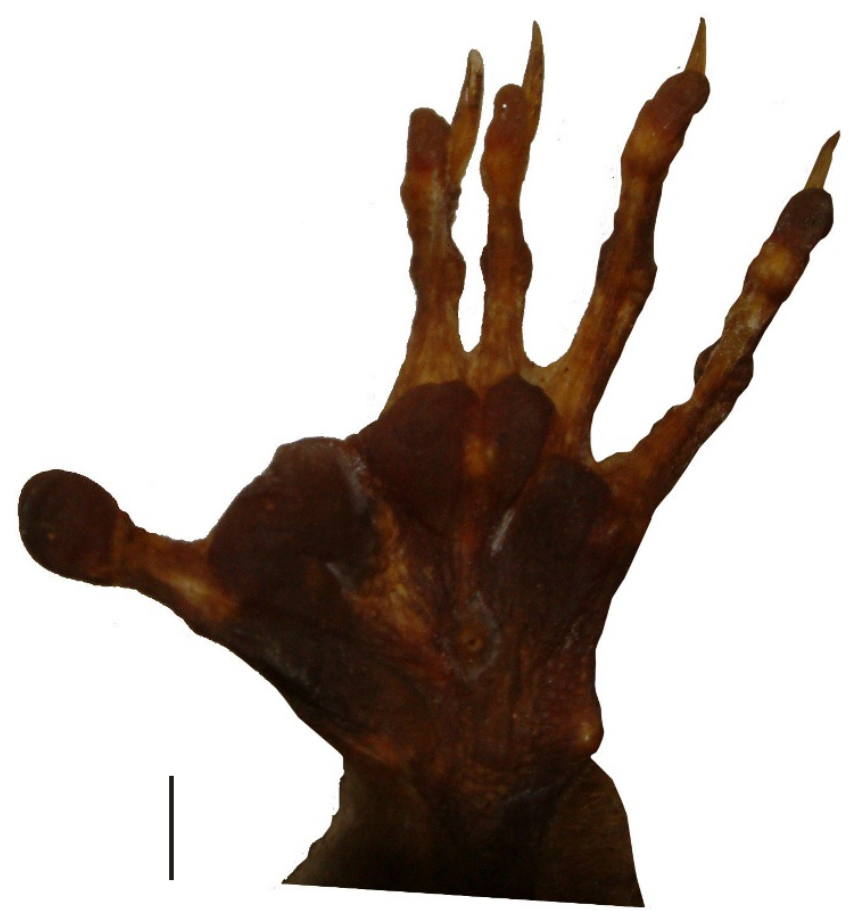

Figure 6

Foot of Caluromys derbianus. Palmar view of the foot of the didelphimorph Caluromys derbianus showing shorter, unwebbed digits II and III; this occurs in some didelphimorphs and has been considered "incipient syndactyly". However, these digit proportions are common in arboreal mammals and do not seem equivalent to syndactyly. Scale bar $=1 \mathrm{~cm}$

Between-digit ratio differences also reveal considerable differentiation of digits with respect to each other (except II and III) according to locomotion. Although not morphometrically assessed, digit I also follows widely recognized patterns of functional adaptation [52]: it is strongly developed and opposable in the arboreal possums and koalas, and reduced or absent in the hopping or bounding kangaroos and bandicoots, which is consistent with patterns of marginal digit loss observed in cursorial, bounding and hopping mammals $[37,52,56]$.

The fact that syndactylous digit proportions differ significantly between locomotor groups supports the hypothesis that they evolve as a unit subject to selection pressures. This complements the results of dissection studies which show that the muscles operating the syndactylous digits are as well developed and functional as in non-syndactylous species [summarized in [3,33]]. In this respect, length similarity of syndactylous phalangeal elements may hold an important implication for their mobility: because the digits are tightly appressed under a common skin sheath and bound by connective tissue [3], their capacity to flex 
and extend depends on alignment of the joints along a plane that allows them to move together.

It is notable that proportions and morphology of all pedal digits in macropodoids (kangaroos) and peramelemorphs are highly similar (see Figure $2 \mathrm{c}, \mathrm{d}$ ). This includes an enlarged digit IV as the main axis of the foot, a slightly smaller digit V, very thin and gracile digits II and III, and reduced or absent digit $\mathrm{I}$. This convergence is interesting because emphasis of central digits often coincides with evolutionary trends in placentals towards cursoriality or bounding locomotion $[37,61]$. Tyndale-Biscoe [62] suggested that syndactyly arose as an adaptation to cursoriality or bounding in Peramelemorpha and Diprotodontia. This is unlikely if it is true that the ancestors of both clades were arboreal $[6,8,16,25,28,36]$. Conversely, however, the functional loss of a digit in syndactylous feet may have favoured the evolution of the bounding or half-bounding habit in both clades.

\section{Syndactyly may not be a functional-adaptive trait}

The few studies that have been concerned with the origins of syndactyly have invoked functional-adaptive scenarios for their appearance, either as grooming organs $[3,11,18,31]$ or as an adaptation to arboreality $[13,28,29]$. However, marsupial syndactyly does not resemble any autopodial adaptations ever evolved in mammalian autopodia. The characteristics of syndactyly are unique among terrestrial mammalian orders (except for three species of otter shrews). The coinciding heterochrony and integration in syndactylous digits also cannot be explained by simple processes of locomotor adaptation, particularly because these patterns are conservative across the considerable diversity of locomotor types in Diprotodontia. Rather, syndactyly may represent a constraint that the foot is adapted to. Under this scenario, digits IV and V, which are always well developed, compensate for the "unification" of the two syndactylous digits. An interesting further implication of viewing syndactyly as a constraint would be a new view of the perceived "reduction" of the syndactylous digits, which has been taken to be a hallmark of syndactyly $[3,13,21,28]$. If digits II and III were constrained to evolve as a single digit and functional unit, it would be expected that the most advantageous dimensions of this complex would be that of a single digit, which is indeed reflected in the dimensions of the syndactylous complex [13]. This would also explain why the much-noted "reduction" is not a general feature of marsupial syndactylous digits. For example, in most arboreal species the syndactylous digits are well developed [[3]; see also Fig. 2a, b]. In fact, the syndactylous digit complex in wombats is longer, although not wider, than digits IV and V (pers. obs.), which is congruent with their digging habit $[56,61]$. As such, there is evidence to suggest that the "reduction" of digits is not an intrinsic property of syndactyly, but may be it's consequence.

\section{Character distribution of syndactyly}

The question as to whether marsupial syndactyly arose once or twice has been discussed extensively, mainly because convergent origins of this perceivedly complex character have been deemed unlikely [16]. A sister group relationship between Peramelemorpha and Diprotodontia, and as such a single origin for syndactyly in these two clades, can be ruled out based on the latest phylogenies. However, it has been suggested that syndactyly may be in fact homoplastic within a larger marsupial clade $[21,23,27]$. Support for this notion comes from possible tracks of a syndactylous marsupial from the Cretaceous of British Colombia [63] and, tentatively, from the Late Cretaceous of Western Colorado [64]. Moreover, claims of "incipient syndactyly" in some South American Didelphimorphia and the marsupial mole Notoryctes typhlops have been considered evidence of an older origin of syndactyly. Extensive connection of digits II and III by skin was only observed in the fully webbed feet of the semiaquatic Yapok Chironectes minimus and the mostly webbed feet of Notoryctes typhlops, but digits II and III and their elements do not resemble each other and are not closely appressed [3]. The sporadic occurrence of digits II and III which are shorter than digit IV in some didelphimorphs is not unusual for mammals. It is neither as highly conserved as syndactyly in Diprotodontia and Peramelemorpha, nor does it resemble it to a great degree. It is possible that the shorter length of digits II and III in these species is due to their mostly arboreal or scansorial habit [49], which commonly coincides with short medial digits $[7,55,65,66]$. The results of this study therefore suggest that syndactyly is convergent in Diprotodontia and Peramelemorpha, and is not plesiomorphic for marsupials.

\section{Scenarios for the origins of syndactyly}

Given the extensive similarities of peramelemorph and diprotodontian syndactyly, the convergent evolution of this trait in the two clades appears unlikely to have occurred at random. It is possible that convergence of syndactyly constitutes a case of parallelism which develops through similar or identical developmental pathways [1]. This would suggest that that there is a greater likelihood of syndactyly evolving in peramelemorphs and diprotodontians due to shared ancestral developmental patterns. Occurrence of full syndactyly in early marsupials and/or metatherians, as has been suggested based on Cretaceous tracks $[63,64]$, is also conceivable under this scenario.

How syndactyly could establish itself twice, and the nature of the developmental changes involved, are difficult to establish in the absence of a functional-adaptive scenario. However, the results of this study provide cir- 
cumstantial evidence that allow the position of hypotheses for further testing. Integration and isometry of digits II and III can be explained in two ways; firstly, as an adaptation to a bounding of digits by skin, and secondly, as the result of a change in ontogenetic patterns that "synchronized" the morphology of the syndactylous digits. Under the first scenario, syndactyly would have established itself as a malformation, possibly during a time when the ancestral populations were undergoing a bottleneck event [13]; the length isometry of the digits could have arisen because this allowed the digits to function as a unit (see above). However, this includes the unparsimonious scenario of a malformation establishing itself in two unrelated marsupial populations, with subsequent highly similar morphological outcomes. Also, if syndactyly were just a skin fusion of the digits, this would not explain the ossification heterochrony that coincides with syndactyly in Diprotodontia and Peramelemorpha.

The second scenario, positing an ontogenetic pattern change, may be more parsimonious and is favoured by some of the evidence from this study. If the morphometric integration of digit elements is intrinsic to syndactyly through an ontogenetic constraint, the establishment of syndactyly as a non-adaptive trait is less improbable because, as discussed above, it effectively retains the capacity of digit flexion and extension. As such, syndactyly would only represent the loss of a single digit, rather than the incapacitation of both digits as in soft-tissue syndactyly of the human hand [67]. Digit loss is common in mammals, although marginal digits are usually affected $[32,36,37,52]$. With functional syndactylous digits, the net loss of a single digit may have had a mild impact, compared to a loss of flexing capacity, as is the case in syndactylous human hands. This may have aided the spread of marsupial syndactyly through the ancestral populations.

Phenotypes resembling marsupial syndactyly in that the digits display identical morphologies have existed in some of the earliest tetrapods such as Acanthostega; this similarity has been ascribed to identical patterns of morphogen expression in the digits [48]. Identical digits have also been created through biochemical manipulation of developing autopodia of mice, chicks, and frogs [68-71]. These experiments largely involve alteration in the expression or concentration of morphogens [mostly bmp family transcription factors; $[68,69,72,73]]$. It is notable that many of these are intimately associated with retention of the inter-digital membrane as it occurs in syndactylous marsupials. As ossification heterochrony and integration of digits II and III hint at ontogenetic changes in these two digits, it should be tested whether the switch to syndactyly involves a naturally occuring change in one of these pathways. Should this be the case, it would be predicted that the ancestral phenotype of syndactyly arose rapidly and was largely similar to that of the more plesiomorphic syndactylous marsupials today. This would provide an avenue for rapid origin of fully integrated, and as such fully functional, syndactylous digits. It would also explain the lack of intermediates of syndactylous feet in extant or extinct marsupials and the heterochrony observed in this study. A similar scenario has been argued by Sears [40] with respect to the origin of bat wings, explaining the conservative size relationship of digits across bat species, as well as the lack of fossil intermediates of bat wings, with a localized change in bmp expression patterns.

\section{Tarsal-metatarsal articulations are not impacted by syndactyly}

The generalized pattern of tarsal-metatarsal articulations throughout marsupials is that digit I articulates with the ectocuneiform, digit II with the mesocuneiform, digit III with the ectocuneiform, and digits IV and V articulate with the cuboid bone. This pattern is highly conserved among marsupials and is already present in the oldest metatherian tarsus known to date, belonging to Sinodelphys szalayi [74]. Woodburne and Case [27] argued that differences of tarsal/metatarsal arrangement in Diprotodontia and Peramelemorpha suggest convergent evolution of syndactyly in these clades. However, the results of this study disagree with Woodburne and Case's [27] reports in several points. Their claim that the endocuneiform is lost in macropodoids cannot be confirmed [see also [25]], and no evidence was found to support a "lateral dislocation" of peramelemorph metatarsals with respect to tarsals. Indeed, no difference in articulation patterns of peramelemorphs compared to other marsupials was noted, other than that pertaining to digit IV. Given the conservatism of tarsal anatomy with respect to metatarsals II and III across all marsupials, it seems that syndactyly is a phenomenon exclusively confined to digit morphology and has no impact on tarsal morphology. The peramelemorph tarsal arrangement also appears in hoofed placentals, where it is considered to represent an adaptation to the emphasis on the central digits [15]. It is noteworthy that kangaroos, whose plesiomorphic locomotion patterns resemble those of peramelemorphs [75], also show a tendency towards increased contact between metatarsal IV and the ectocuneiform. This suggests that the extensive contact between metatarsal IV and the ectocuneiform in peramelemorphs is related to a stabilizing re-arrangement, rather than a result of syndactyly.

\section{Marsupial and human syndactyly}

Marsupial syndactyly derives its name from a congenital malformation in humans, and has been treated as directly comparable $[13,23,42,50,62]$. Human syndactyly is an overarching term for a diverse congenital condition which always involves incomplete digit separation during early development due to a lack of inter-digit membrane cell 
death, or through synostoses [76]. Marsupial syndactyly only corresponds to a mild subtype of syndactyly termed "zygodactyly" $[77,78]$. However, in its worst form human syndactyly can involve synostoses of the majority of digit elements [76]. Contrary to a popular notion in the field of marsupial systematics $[21,23,42,62]$ the genetic background of syndactyly is complicated and only known for a few of the over 50 types $[76,77]$. It is notable that even the mildest forms of syndactyly in the hand lead to incapacitation of grasping capability of the affected digits and require surgical treatment, although they are rarely separated in the foot [67]. Human syndactyly is only partly heritable, and within families where syndactyly is passed on, it manifests variably in terms of severity, the limb affected, and number of digits involved [77]. The present results reveal that marsupial syndactyly is a highly specific phenotype that does not compare to human syndactyly more than superficially because marsupial syndactyly is entirely heritable and highly conservative in its manifestation. As such, the term "syndactyly" is slightly unfortunate for the unique condition found in marsupials, and has in the past lead to the uncritical assumption that the two conditions are directly comparable. It may be desirable to clearly distinguish between the two conditions by consistently referring to marsupial syndactyly as such, or introducing a new term (for example, "homodactyly" may be appropriate given the similarity of digits II and III) altogether.

\section{Conclusion}

The diverse methods employed in this study have provided novel insights on the phenomenon of marsupial syndactyly. The results demonstrate that the syndactylous digits of marsupials evolve as a unit which is subject to functional adaptation like the remaining digits of the foot. The strong integration between the two digits may be due to a change in early developmental patterning, as the ossification heterochrony between syndactylous compared to non-syndactylous species suggests. No evidence was found for incipient syndactyly in other marsupials, but the ossification similarity of pedal digits in Diprotodontia and Peramelemorpha suggest an underlying developmental parallelism. We argue that locomotor adaptation is not likely to be the cause for syndactyly; based on our results, we suggest a scenario in which syndactyly arose as a single change in digit ontogeny that amounted to the loss of a single digit, rather than incapacitation of both digits through skin webbing. We also show that syndactyly is restricted to Peramelemorpha and Diprotodontia, and has no influence on tarsal bone arrangement in either clade. Together, the results change the relevance of marsupial syndactyly from being an enigmatic phylogenetic character within marsupials to a rare transformation of digit morphology whose molecular background has the potential to provide important insights into the patterns of digit evolution.

\section{Methods \\ Morphometric data collection}

Articulated pedal skeletons from specimens of 32 syndactylous marsupial species were measured. Species were selected to include the full range of pedal diversity in syndactylous marsupials. Raw measurements and accession numbers can be found in Additional files 1 and 4; for terminology, refer to Figure 2a. Measurements of 11 phalangeroid and petauroid possums, 11 kangaroos, 7 vombatiforms (four extinct), and 4 peramelemorphs were taken using digital calipers (specified accuracy \pm 0.02 $\mathrm{mm}$ ). In most extant species, two adult specimens per species were measured and measurements were averaged following Christiansen [79]; all individual measurements were taken twice and averaged. Measurements comprised length and mid-shaft width of metatarsals, proximal and intermediate phalanges II-V (a total of 6 measurements per digit). Measurements for digit I were not taken since this digit is reduced or lost in kangaroos and bandicoots.

\section{Matrix comparisons and assessment of raw measurements of non-size adjusted data}

To assess inter-digit integration patterns across the diversity of syndactylous marsupials, a variance/covariance and correlation matrix comparison approach was employed. Matrix comparisons are suited for this problem because they can quantify integration patterns that may constrain morphological variability of the trait components with respect to each other [58,80-83]. Natural logarithm-transformed morphometric measurements were investigated. The measurements were not otherwise adjusted by size because relative size variation is an important factor in differences between the syndactylous and non-syndactylous digits. Correlation and variance/covariance matrices of digit measurements were computed for each digit, resulting in $6 \times 6$ matrices. Correlation matrices were compared using a Mantel's test [84] implemented by the freeware Microsoft Excel-addin PopTools [85], which compares the matrices and provides a non-parametric estimate for the significance of the correlation. Variance-covariance matrices were compared using the random skewers method $[82,86]$, run through a Monte-Carlo simulation implemented by PopTools [58]. Matrix repeatabilities were also computed as a measure of sampling error following Cheverud [80] and Marroig and Cheverud [82] using the Monte-Carlo simulation routine in PopTools.

Matrix correlations provide an estimate of correlated evolution between the traits studied, but they do not provide information on absolute size relationships. In other words, two anatomical complexes can have highly similar matrices but be of greatly differing absolute sizes. As 
noted above, size relationships between digits are a crucial component in this study because syndactyly manifests itself in unusually "synchronized" - looking size relationships between digits II and III. To address this, raw length measurements were plotted for visual assessment and their correlations were compared using Fisher's z test [87].

\section{Locomotor indices - proportional differences}

Morphometric correlates of locomotion of non-syndactylous autopodial anatomy are well understood $[7,52]$, so that it is possible test whether the syndactylous foot is subject to generally recognized patterns of locomotor adaptation. The species measured were divided into three locomotor groups: hopping and bounding species (kangaroos/rat kangaroos and bandicoots), arboreal species (phalangeroid and petauroid possums), and terrestrial plantigrade species (vombatiforms). Based on the morphometric measurements, differences in morphometric locomotor predictor indices were computed. Using ratiobased locomotor indices is a convenient way of focusing on that part of the variation which is explained exclusively by proportional, rather than size-related, differences. The phalangeal index [proximal and intermediate phalangeal length as a percentage of metatarsal length; [59,88]] and metatarsal, proximal phalangeal, and intermediate slenderness ratios [SRs; element length*100/element width; [7]] were employed because they are known to be reliable proxies of locomotor habit. Locomotor index comparisons were conducted on two levels: Firstly, measurements in single digits were compared between locomotor groups. Secondly, ratios between locomotor indices of digit pairs were compared to capture some of the disparities in between-digit proportions in species of different locomotor types.

Because of disparate and relatively small sample sizes in each locomotor group, the non-parametric Kruskal-Wallis test was performed to assess whether significant differences existed within the three locomotor groups. Indices for which significant results were found were also investigated using post-hoc Wilcoxon signed rank sum tests to assess whether all three locomotor types are distinguished using the predictor indices.

\section{Digit ossification sequences}

To investigate pedal digit ontogeny in syndactylous marsupials, ossification sequences of pedal digit elements were recorded for ontogenetic series of 5 diprotodontian species, the peramelemorph Isoodon macroura, 2 dasyurids, the ameridelphian Didelphis virginiana, the placental mouse Mus musculus, and the skincid lizard Hemiergis peronii as an outgroup. Museum accession numbers are listed in Additional file 4. Sequence data were collected from the literature for Hemiergis peronii [89], Mus musculus [54], Sminthopsis macroura [90], and Didelphis virginiana
$[91,92]$. Pouch young from the personal collection of VW were clear stained according to Dingerkus and Uhler [93], modified by Prochel [94]. The remaining specimens were investigated by acquiring X-ray images (using a SkyScan 1172 desktop micro-computer tomography scanner) of the specimen's lower half of the body, taken at $30^{\circ}$ intervals during a $360^{\circ}$ rotation to allow a multi-angle view of the foot.

The ossification onset of metacarpals and phalangeal elements in all digits was recorded. For each species, ranks were given to ossifications in the order in which they occur, and compared across species. Sequence heterochrony in the appearance of ossification sequences was also summarized by coding the sequences into event pairs $[46,95]$ and analysing these using the Parsimov program package [96]. The consensus of ACCTRAN and DELTRAN optimized analyses is presented here. This means that ambiguous transformations are not considered, which results in a conservative but more reliable estimate [96]. Because the sample for peramelemorphs contained only one poorly resolved species, Parsimov was run without it.

Tarsal-metatarsal articulations and "incipient syndactyly" The tarsal-metatarsal joint was investigated in 23 species from 5 marsupial orders. Accession numbers can be found in Additional file 4; for terminology, refer to Figure 2a. When possible, several individuals per species were investigated. The tarsal/metatarsal articulations were listed for each species.

A literature search for reports of "incipient syndactyly" was conducted, and species for which reports exist were re-investigated (Table 1). This was done using study skins whose pedal skeleton was left in the skin, and articulated pedal skeletons (for accession numbers, see Additional file 4).

\section{Authors' contributions}

VW wrote the manuscript, collected and analysed the morphometric data and most ossification data except those obtained from clearing and staining. MN conducted the clearing and staining on specimens provided by VW and collected and analysed data on tarsal morphology and incipient syndactyly. $\mathrm{MN}$ also reviewed the literature on the historical, phylogenetic, and molecular context of syndactyly and contributed to the drafting of the manuscript. Both authors read and approved the final manuscript. 


\section{Additional material}

Additional file 1
Raw morphometric data. Raw morphometric data
Click here for file
[http://www.biomedcentral.com/content/supplementary/1471-
$2148-8-160-S 1 . x l s]$
Additional file 2
Kruskal-Wallis and Wilcoxon rank-sum test results for differences in loco-
motor indices. Rank-sum test results for differences in locomotor indices.
Click here for file
[http://www.biomedcentral.com/content/supplementary/1471-
$2148-8-160-S 2 . d o c]$
Additional file 3
Ossification data. Ranks of timing, from earliest to latest, for the species
considered in the analysis of pedal digit ontogeny.
Click here for file
[http://www.biomedcentral.com/content/supplementary/1471-
$2148-8-160-S 3 . d o c]$
Additional file 4
Accession numbers. Accession numbers or sources for investigations
related to ossification sequence, tarsal/metatarsal, and "incipient syndac-
tyly".
Click here for file
[http://www.biomedcentral.com/content/supplementary/1471-
$2148-8-160-S 4 . d o c]$

\section{Acknowledgements}

We wish to thank M. Sánchez-Villagra for generous help and advice with this manuscript, and for hosting MNs research visit at the NHM. Thanks also to S. Ingleby (Australian museum), C. Kemper and D. Stemmer (South Australia Museum) for making pouch young specimens available, and Allan Jones for technical advice on CT scanning. We thank R. Voss (American Museum of Natural History), D. Hills and R. Sabin (Natural History Museum, London) for access to Museum collections. C. Leigh from the Anatomical Institute, Adelaide University, and the Marsupial Immunology Group, Macquarie University, generously provided specimens for clearing and staining. For discussion and advice we thank K. Sears, M. Archer, R. Beck, O. Bininda-Emonds, A. Goswami, S. Hand, A. Janke, J. Louys, K. Monro and T. Säll. Two anonymous reviewers provided valuable comments that improved the manuscript. We thank the Solander Program for funding a collaborative visit of $\mathrm{VW}$ to Lund. MN acknowledges financial support of the European Commission's Research Infrastructure Action through the SYNTHESYS Project (GB-TAF-1339) to visit the Natural History Museum, London. VWs work was funded by a University Internal Postgraduate Award (UIPA) of the University of New South Wales, and use of the CT scanner was funded through a transport and access grant of the Australian Nanostructural Analysis Network Organisation.

\section{References}

I. Hall BK: Homoplasy and homology: Dichotomy or continuum? Journal of Human Evolution 2007, 52:473-479.

2. Hennig W: Grundzüge einer Theorie der phylogenetischen Systematik. Berlin, Deutscher Zentralverlag; 1950:370.
3. Müller J: Die Mechanik der syndactylen Zehen von Macropus und anderen Beuteltieren und ihre Verwendung als Putzorgan. Zoomorphologie 1930, 17:154-218.

4. Owen R: On Hypsiprymnodon, Ramsay, a genus indicative of a distinct family (Pleopodidae) in the diprotodont section of the Marsupialia. Transactions of the Linnean Society, 2nd Series 1878, I:573-582.

5. Wilson DE, Reeder DM: Mammal Species of the World. Baltimore , Johns Hopkins University Press; 2005:2 I42.

6. Aplin KP, Archer M: Recent advances in marsupial systematics with a new syncretic classification. In Possums and Opossums Studies in Evolution Volume I. Edited by: Archer M. Sydney, Surrey Beatty \& Sons and the Royal Zoological Society of New South Wales; 1987:xv-Ixxii.

7. Weisbecker V, Warton DI: Evidence at Hand: diversity, functional implications, and locomotor prediction in intrinsic hand proportions of diprotodontian marsupials. Journal of Morphology 2006, 267:|469-| 485.

8. Bensley BA: On the evolution of the Australian Marsupialia: with remarks on the relationships of the marsupials in general. Transactions of the Linnean Society of London, Zoology 1903, 2:83-217.

9. Gervais P: Mammalogie ou Mastologie. In Dictionnaire pittoresque d'Histoire Naturelle et des Phenomènes de la Nature Volume 4. Edited by: Guérin-Meneville FE. Paris ; 1836:614-640.

10. Gregory WK: The orders of mammals. Bulletin of the American Museum of Natural History 1910, 27: I-524.

II. Jones FW: The Mammals of South Australia. Adelaide, British Science Guild; 1924:137.

12. Ride WDL: On the evolution of Australian marsupials. In The Evolution of Living Organisms Edited by: Leeper GW. Melbourne , Melbourne University Press ; 1962:28I-306.

13. Hall LS: Syndactyly in marsupials-problems and prophecies. In Possums and Opossums Volume I. Edited by: Archer M. Sydney, Surrey Beatty and Sons; 1987:245-255.

14. Luckett WP: Suprafamilial relationships within Marsupialia: resolution and discordance from multidisciplinary data. Journal of Mammalian Evolution 1994, 2:225-283.

15. Marshall LG: Evolution of the peramelid tarsus. Proceedings of the Royal Society of Victoria 1972, 85:5I-60.

16. Szalay F: Evolutionary history of the marsupials and of osteological characters. New York, Cambridge University Press; 1994.

17. Mickoleit G: Phylogenetische Systematik der Wirbeltiere. Munich, Verlag Dr. Friedrich Pfeil; 2004:671.

18. Abbie AA: Some observations on the major subdivisions of the Marsupialia with special reference to the position of the Peramelidae and Caenolestidae. Journal of Anatomy 1937, 7I:429-435

19. Baker ML, Wares JP, Harrison GA, Miller RD: Relationships among the families and orders of marsupials and the major mammalian lineages based on recombination activating gene- I. Journal of Mammalian Evolution 2004, I I: I I-I6.

20. Amrine-Madsen HM, Scally M, Westerman M, Stanhope MJ, Krajewski $C$, Springer MS: Nuclear gene sequences provide evidence for the monophyly of australidelphian marsupials. Molecular phylogenetics and evolution 2003, 28: 186-196.

21. Kirsch JAW: The comparative serology of Marsupialia, and a classification of marsupials. Australian Journal of Zoology Supplementary Series 1977, 52: I-I 52.

22. Nilsson MA, Arnason U, Spencer PBS, Janke A: Marsupial relationships and a timeline for marsupial radiation in South Gondwana. Gene 2004, 340:189-196.

23. Phillips MJ, McLenachan PA, Down C, Gibb G: Combined mitochondrial and nuclear DNA sequences resolve the interrelations of the major Australasian marsupial radiation. Systematic Biology 2006, 55: 122-137.

24. Asher RJ, Horovitz I, Sánchez-Villagra MR: First combined cladistic analysis of marsupial phylogenetic relationships. Molecular Phylogenetics and Evolution 2004, 33:240-250.

25. Horovitz I, Sánchez-Villagra MR: A morphological analysis of marsupial mammal higher - level phylogenetic relationships. Cladistics 2003, 19:18|-|2|2.

26. Baverstock PR, Birrell J, Krieg M: Albumin immunologic relationships among Australian possums: a progress report. In Possums and Opossums: Studies in Evolution Edited by: Archer M. Chipping 
Norton, New South Wales, Australia., Surrey Beatty and Sons; 1987:229-234

27. Woodburne MO, Case JA: Dispersal, Vicariance, and the late Cretaceous to early Tertiary land mammal biogeography from South America to Australia. Journal of Mammalian Evolution |996, 3:|2|-|6|.

28. Dollo L: Les ancêtres marsupiaux étaient-ils arboricoles? Miscellanées biologiques 1899, Travaux du Station Zoologique Wimereux: $188-203$

29. Szalay F: A new appraisal of marsupial phylogeny and classification. In Carnivorous Marsupials Edited by: Archer M. Sydney, Royal Zoological Society; 1982:62 I-640.

30. Goodrich ES: Syndactyly in marsupials. Proceedings of the Zoological Society of London 1935:175-178.

31. Owen R: On the osteology of the Marsupialia. Transactions of the Zoological Society of London 1874, 8:483-500.

32. Shubin N: Origin of Evolutionary Novelty: Examples from Limbs. Journal of Morphology 2002, 252:15 -28.

33. Barbour RA: The musculature and limb plexuses of Trichosurus vulpecula. Australian Journal of Zoology 1963, I I:488-610.

34. Carlsson A: Über Dendrolagus dorianus. Zoologische Jahrbücher 1914, 36:547-620

35. Carlsson A: Zur Morphologie des Hypsiprymnodon moschatus. Kungelige Svenska Vetenskapsakademiens Handlingar 1915, 52: I-5I.

36. Bensley BA: On the question of an arboreal ancestry of the Marsupialia, and the interrelationships of the mammalian subclasses. American Naturalist 1901, 35: I 17-137.

37. Lull RS: Adaptations to aquatic, arboreal, fossorial and cursorial habits in mammals. IV. Cursorial Adaptations. The American Naturalist 1904, 38: I-I I.

38. Shubin N, Tabin C, Carroll S: Fossils, genes and the evolution of animal limbs. Nature 1997, 388:639-648.

39. Gould S): Ontogeny and Phylogeny. Cambridge, MA , Harvard University Press; 1985:520.

40. Sears K, Behringer RR, Rasweiler J], Niswander LA: Development of bat flight: Morphologic and molecular evolution of bat wing digits. Proceedings of the National Academy of Science, USA 2006, 103:658|-6586.

41. Wagner GP, Chiu C, Laubichler M: Developmental evolution as a mechanistic science: the inference from developmental mechanisms to evolutionary processes. American Zoologist 2000, 40:819 -831.

42. Kirsch JAW, Lapointe FJ, Springer MS: DNA - hybridisation studies of marsupials and their implications for metatherian classification. Australian Journal of Zoology 1997, 45:2 II-280.

43. Simpson GG: The Argyrolagidae, extinct South American marsupials. Bulletin of the Museum of Comparative Zoology 1970 139: |-86.

44. Bininda-Emonds ORP, Jeffery JE, Coates MI, Richardson MK: From Haeckel to event-pairing: the evolution of developmental sequences. Theory in Biosciences 2002, 1 $21: 297-320$.

45. Sánchez-Villagra MR: Comparative patterns of postcranial ontogeny in therian mammals: an analysis of relative timing of ossification events. J Exp Zool 2002, 294:264-273.

46. Smith KK: Sequence heterochrony and the evolution of development. Journal of Morphology 2002, 252:82-97.

47. Begin M, Roff DA: From micro-to macroevolution through quantitative genetic variation: positive evidence from field crickets. Evolution 2004, 58:2287-2304.

48. Tabin C): Why we have (only) five fingers per hand: Hox genes and the evolution of paired limbs. Development 1992 I 1 6:289-296.

49. Nowak RM: Walker's Mammals of the World. Volume I. 6th edition. Baltimore, The Johns Hopkins University Press; 1999:2015.

50. Straus WLJ: The nature and inheritance of webbed toes in man. Journal of Morphology 1926, 41:427-439.

51. Lynch JD: Apparent syndactyly in a Mexican frog. Journal of the Ohio Herpetological Society 1965, 5:57.

52. Hildebrand M, Goslow G: Analysis of vertebrate structure. 5th edition. New York, John Wiles \& Sons; 2001:635.

53. Shubin N, Alberch P: A morphogenetic approach to the origin and basic organization of the tetrapod limb. Evolutionary Biology 1986, 20:319-387.
54. Johnson ML: The time and order of appearance of ossification centers in the albino mouse. The American Journal of Anatomy 1933, 52:24|-27|.

55. Jouffroy FK, Godinot M, Nakano Y: Biometrical characteristics of primate hands. In Hands of Primates Edited by: Preuschoft H, Chivers DJ. Wien , Springer; 1993:133-17|.

56. Weisbecker V, Schmid S: Autopodial skeletal diversity in hystricognath rodents: functional and phylogenetic aspects. Mammalian Biology 2007, 72:27-44.

57. Capdevila J, Belmonte JCl: Perspectives on the evolutionary origin of tetrapod limbs. Journal of Experimental Biology 2000, 288:287 $-303$.

58. Young NM, Halgrímsson: Serial homology and the evolution of the mammalian limb covariation structure. Evolution 2005 , 59:269I-2704.

59. Lemelin P: Morphological correlates of substrate use in didelphid marsupials: implications for primate origins. Journal of Zoology, London 1999, 247:165-175.

60. Taylor ME: The functional anatomy of the forelimb of some African Viverridae (Carnivora). Journal of Morphology 1974, I 43:307-336.

6I. Hildebrand M: Digging of quadrupeds. In Functional Vertebrate Morphology Edited by: Hildebrand M, Bramble DM, Liem KF, Wake DB. Cambridge, MA, Belknop; 1985:89-109.

62. Tyndale-Biscoe: Life of Marsupials. Collingwood, Australia CSIRO Publishing; 2005:320.

63. Sarjeant WA: Probable marsupial footprints from the Cretaceous sediments of British Columbia. Canadian Journal of Earth Sciences 1986, 23: 1 223-1227.

64. Lockley MG, Foster JR: Late Cretaceous mammal tracks from North America. Ichnos 2003, 10:269-276.

65. Cartmill M: Pads and claws in arboreal locomotion. In Primate Locomotion Edited by: Jenkins FA. New York, Academic Press; 1974.

66. Weisbecker V, Sánchez-Villagra MR: Carpal evolution in diprotodontian marsupials. Zoological Journal of the Linnean Society 2006, 146:369-384.

67. Skinner HB: Current Diagnosis \& Treatement in Orthopedics. 4th edition. New York, McGraw-Hill Professional; 2006.

68. Dahn RD, Fallon JF: Interdigital regulation of digit identity and homeotic transformation by modulated bmp signaling. Science 2000, 289:438-44I.

69. Suzuki T, Takeuchi J, Koshiba-Takeuchi K, Ogura T: Tbx genes specify posterior digit identity through Shh and BMP signaling. Developmental Cell 2004, 6:43-53.

70. Wang B, Fallon JF, Beachy PA: Hedgehog-regulated processing of Gli3 produces an anterior/posterior repressor gradient in the developing vertebrate limb . Cell 2000, 100:423-434

7I. Verheyden JM, Lewandoski M, Deng C, Harfe BD, Sun X: Conditional inactivation of Fgfr I in mouse defines its role in limb bud establishment, outgrowth and digit patterning. Development 2005, I 32:4235-4245.

72. Bandyopadhyay A, Tsuji K, Cox K, Harfe BD, Rosen V, Tabin CJ: Genetic analysis of the roles of BMP2, BMP4, and BMP7 in limb patterning and skeletogenesis. Public library of Science Genetics 2006, 2:2116-2130

73. Weatherbee SD, Behringer RR, Rasweiler JIIV, Niswander LA: Interdigital webbing retention in bat wings illustrates genetic changes underlying amniote limb diversification. Proceedings of the National Academy of Science, USA 2006, 103:15103-15107.

74. Luo ZX, Ji Q, Wible JR, Yuan CX: An early Cretaceous tribosphenic mammal and metatherian evolution. Science 2003 , 302: $1934-1940$

75. Burk A, Westerman M, Springer MS: The phylogenetic position of the musky rat-kangaroo and the evolution of bipedal hopping in kangaroos. Systematic Biology 1998, 47:457-474.

76. Malik S, Ahmad W, Grzeschik KH, Koch MC: A simple method for characterising syndactyly in clinical practice. Genet Couns 2005, 16(3):229-238.

77. Malik S, Schott J, Ali SW, Oeffner F, Amin-ud-Din M, Ahmad W, Grzeschik KH, Koch MC: Evidence for clinical and genetic heterogeneity of syndactyly type I: the phenotype of second and third toe syndactyly maps to chromosome 3p2 I.3 I. European Journal of Human Genetics 2005, 13:1268-1274.

78. Weidenreich F: Die Zygodaktylie und ihre Vererbung. Zeitschrift für induktive Abstammungs-und Vererbungslehre 1923, 32:304-309. 
79. Christiansen P: Scaling of mammalian long bones: small and large mammals compared. Journal of Zoology, London 1999, 247:333-348.

80. Cheverud JM: Quantitative genetic analysis of cranial morphology in the cotton-top (Saguinus oedipus) and saddleback (S. fuscicollis) tamarins. Journal of Evolutionary Biology 1996, 9:5-42.

81. Goswami A: Morphological integration in the carnivoran skull. Evolution 2006, 60:169-183.

82. Marroig G, Cheverud JM: A comparison of phenotypic variation and covariation patterns and the role of phylogeny, ecology, and ontogeny during cranial evolution of New World monkeys. Evolution 200I, 55:2576-2600.

83. Steppan SJ: Phylogenetic analysis of phenotypic covariance structure. I. Contrasting results from matrix correlation and common principal component analyses. Evolution 1997, $5 \mathrm{I}: 57 \mathrm{I}-586$.

84. Mantel NA: The detection of disease clustering and a generalized regression approach. Cancer Research 1967, 27:209-220.

85. Hood GM: PopTools V. 2.6.9. 2005 [http://www.cse.csiro.au/pop tools].

86. Cheverud JM: Phenotypic, genetic, and environmental integration in the cranium. Evolution 1983, 36:499-516.

87. Fisher RA: On the probable error of a coefficient of correlation deduced from a small sample. Metron I92I, I:I-32.

88. Napier JR: Prehensility and opposability in the hands of primates. Symposium of the Zoological Society, London I96I, 5: I I5-133.

89. Shapiro MD, Hanken J, Rosenthal N: Developmental basis of evolutionary digit loss in the Australian lizard Hemiergis. Journal of Experimental Zoology Part B Molecular and developmental evolution 2003, 297:48-56.

90. Frigo L, Wooley PA: Development of the skeleton of the stripefaced dunnart, Sminthopsis macroura (Marsupialia: Dasyuridae). Australian Journal of Zoology 1996, 44:155-164.

91. de Oliveira CA, Nogueira JC, Bohórquez Mahecha GA: Sequential order of appearance of ossification centers in the opossum Didelphis albiventris (Didelphidae) skeleton during development in the marsupium. Annals of Anatomy 1998, 180:113-121.

92. Nesslinger $\mathrm{CL}$ : Ossification centers and skeletal development in the postnatal virginia opossum. Journal of Mammalogy 1956, 37:382-394.

93. Dingerkus G, Uhler LD: Enzyme clearing of alcian blue stained whole small vertebrates for demonstration of cartilage. Stain Technology 1977, 52:229-232.

94. Prochel J: Early skeletal development in Talpa europaea, the common European mole. Zoological Science 2006, 23:427-434.

95. Smith KK: Comparative patterns of craniofacial development in eutherian and metatherian mammals. Evolution 1997, 5I:1663-1678.

96. Jeffery JE, Bininda-Emonds ORP, Coates MI, Richardson MK: A new technique for identifying sequence heterochrony. Systematic Biology 2005, 54:230-240.

Publish with Bio Med Central and every scientist can read your work free of charge

"BioMed Central will be the most significant development for disseminating the results of biomedical research in our lifetime. "

Sir Paul Nurse, Cancer Research UK

Your research papers will be:

- available free of charge to the entire biomedical community

- peer reviewed and published immediately upon acceptance

- cited in PubMed and archived on PubMed Central

- yours - you keep the copyright

Submit your manuscript here:

http://www.biomedcentral.com/info/publishing_adv.asp
BioMedcentral 